\title{
Cu NMR Study of Detwinned Single Crystals of Ortho-II YBCO6.5
}

\author{
Z. Yamani, W.A. MacFarlane Hil, B.W. Statt \\ Department of Physics, University of Toronto, 60 St. George St., Toronto, ON, Canada M5S 1A7 \\ D. Bonn, R. Liang, W.N. Hardy \\ Department of Physics and Astronomy, University Of British Colombia, \\ 6224 Agricultural Road, Vancouver, BC, Canada V6T $1 Z 1$
}

(Dated: November 13, 2018)

\begin{abstract}
Copper NMR has been used as a local probe of the oxygen ordering in Ortho-II $\mathrm{YBa}_{2} \mathrm{Cu}_{3} \mathrm{O}_{6.5}$ crystals grown in $\mathrm{BaZrO}_{3}$ crucibles. Line assignments have been made to each of the expected crystallographically inequivalent sites. The presence of distinct and narrow lines for these sites as well as the lack of a line known to be associated with oxygen defects indicates that these crystals are highly stoichiometric. Our estimate of the lower limit on the chain length is consistent with that derived from $\mathrm{X}$-ray diffraction measurements. In addition, we have found no evidence for static magnetic moments, in contrast to some previous results.
\end{abstract}

\section{INTRODUCTION}

Since the discovery [1] of high temperature superconductors (HTSC), considerable effort has been devoted to understanding the electronic and magnetic properties of these materials at a microscopic level. Although there are a large number of perovskite HTSC materials, the $\mathrm{YBa}_{2} \mathrm{Cu}_{3} \mathrm{O}_{6+x}(\mathrm{YBCO} 6+x)$ family has a special place. It was the first superconductor discovered [2] with a transition temperature $T_{c}$ above liquid nitrogen temperature with a relatively simple synthesis route. It is available in almost the entire region of the phase diagram, easily tuned by varying the oxygen content in a quasi-continuous way. Thus it is appealing for probing the intrinsic properties of HTSCs. In the underdoped regime, a variety of crossover phenomena are observed at temperatures above $T_{c}$ in which various forms of spectral weight at low energies are suppressed. These phenomena are associated with the opening of a pseudogap 3 . To help elucidate the nature of this pseudogap, it is desirable to have highly stoichiometric crystals with a high degree of crystalline order. In particular, such samples are needed if one wants to determine whether or not the crossover behaviors are transistions smeared by disorder and/or impurities [4].

$\mathrm{YBCO} 6+x$ can be prepared in the whole range of oxygen content $6+x=6.0$ to slightly larger than 7.0. The oxygen content affects the crystallographic structure of the material[5]. At $x=1.0$, the crystal structure consists of a bilayer of two-dimensional (2D) $\mathrm{CuO}_{2}$ planes and a single layer of one-dimensional (1D) $\mathrm{Cu}-\mathrm{O}-\mathrm{Cu}$ chains, hereafter denoted the $\mathrm{CuO}$ chain layer. Nuclear magnetic resonance (NMR) spectroscopy offers a unique probe to investigate the microscopic properties of the $\mathrm{CuO}_{2}$ planes and $\mathrm{CuO}$ chain layers separately. In particular, the response of inequivalent sites can in principle be resolved in NMR spectra and the dynamical prop-

*e-mail statt@physics.utoronto.ca erties of these sites studied by nuclear relaxation. The chains are important not only because of their role as charge reservoirs and their proximity to the superconducting $\mathrm{CuO}_{2}$ planes, but also because of their low dimensionality. It is well known that charge/spin density wave(CDW/SDW) ground states are prevalent in quasione dimensional systems []. Therefore, the chains in this system present an important opportunity for clarifying the question of whether such transitions exist in HTSC. In fact this has been the subject of study in the stoichiometric compound $\mathrm{YBa}_{2} \mathrm{Cu}_{4} \mathrm{O}_{8}$ containing two $\mathrm{CuO}$ chain layers, where evidence for the presence of incommensurate CDW fluctuations, near the wave vector $2 k_{F}$ occurring below $180 \mathrm{~K}$ has been reported 7 . There are a few reports on charge modulations in YBCO7 as well [8]. Perhaps the most striking evidence for charge oscillations in the chains of YBCO7 is provided by STM observations 9 .

The structural phase diagram of $\mathrm{YBCO} 6+x$ has been studied in great detail; early neutron diffraction reveal [10] a tetragonal to orthorhombic phase transition as the sample is cooled from the growth temperature, in which oxygens form $1 \mathrm{D} \mathrm{CuO}$ chains and thus break the tetragonal symmetry. Typically the chain ordering occurs in domains and the samples need to be mechanically detwinned in order to produce crystals with a single orientation of chains.

Depending on the preparation conditions and oxygen content, oxygen atoms arrange themselves in different types of ordering in the $\mathrm{CuO}$ chain layers. Efforts to produce crystals with domains of long chains requires an understanding of oxygen ordering in the chain layer. In addition to the orthorhombic structure (Ortho-I, full chains) at $x=1.0$ (Fig. 1 (ii)) and tetragonal phase (T, empty chains) at $x=0.0$ (Fig. 1 (iii)), at least four more modified orthorhombic structures have been observed 11] such as Ortho-II (Fig. 1 (i)) for $6.35 \leq 6+x \leq 6.62$ with alternating full and empty chains. There have been theoretical studies based on lattice gas models to explain the structural phase diagram of $\mathrm{YBCO} 6+x[12]$. These models can be shown to be equivalent to the Ising model with asymmetric next nearest neighbor interac- 
tions (ASYNNNI model) which use three pair potentials between oxygen atoms in $\mathrm{CuO}$ chain layers: $V_{1}$ for nearest oxygen neighbor pairs, $V_{2}$ for next nearest pairs connected through a $\mathrm{Cu}$ ion and $V_{3}$ for those without $\mathrm{Cu}$ in between, with the condition $V_{2}<0<V_{3}<V_{1}$. It is the strongly repulsive interaction $V_{1}$ of two inter-chain nearest neighbor oxygen ions which prevents the occupation of nearest neighbor oxygen sites and promotes the formation of chains. Long chain segments are induced by the attractive potential $V_{2}$ between two intra-chain next nearest neighbor oxygen ions connected through a $\mathrm{Cu}$ ion. Alternating sequences of full and empty chains follow from the weak repulsive $V_{3}$ between two inter-chain next nearest neighbor oxygen ions.

In YBCO6+x, $T_{c}$ changes from the maximum 92.5 $\mathrm{K}$ at optimal doping to zero for fully oxygen depleted samples 13. This change takes place in a nonlinear manner with a pronounced plateau at $60 \mathrm{~K}$ for $6.45 \leq 6+x \leq$ 6.65 and a broad maximum at $92.5 \mathrm{~K}$ for $6.85 \leq 6+x \leq$ 7.0. These features are associated with the Ortho-II and Ortho-I ordered phases, respectively. It is suggested [14] that this correlation has to do with charge transfer rather than with any specific $T_{c}$ of the ordered phases. The importance of oxygen ordering in the $\mathrm{CuO}$ chain layers in determining the charge transfer was first noted in a study of the time dependence of $T_{c}$ during room temperature annealing of quenched samples 15]. With the oxygen content (average occupancy of different oxygen sites) remaining constant, the degree of oxygen ordering increased with time. This resulted in an increase in the orthorhombicity and in $T_{c}$. It was shown that the rearrangement of oxygen ions in the chain layers to form longer chain segments was responsible for this increase. Another experimental indication of the presence of a minimum chain length for the charge transfer to take place is the $T_{N}$ vs. oxygen content for small $x: T_{N}$ is nearly constant for $6+x \leq 6.2[16]$. The relationship between oxygen ordering and hole concentration has been addressed 17] theoretically by band structure calculations. Chain fragments were found to be unable to transfer charge to the planes until they exceeded a critical length equal to three (containing three $\mathrm{Cu}$ and two $\mathrm{O}$ atoms). An alternative model combining the ASYNNNI and the charge transfer models has also been [18] successful in explaining the variation of $T_{c}$ vs. $x$. Here it is assumed that only the ordered phase participates in superconductivity. Thus there is a minimum domain size below which no charge transfer can take place.

NMR experiments can be used to measure the electric field gradient (EFG) in materials. Since the EFG at a nucleus depends on the symmetry of that site, the valence of the ion itself and the charge of its environment, valuable information about the electronic structure of the system under study can be obtained by such measurements; i.e. charge transfer. However, the interpretation can be complicated if the samples are not single phase or suffer from impurities and/or disorder. There are a number of NMR reports on underdoped YBCO6+x with different oxygen

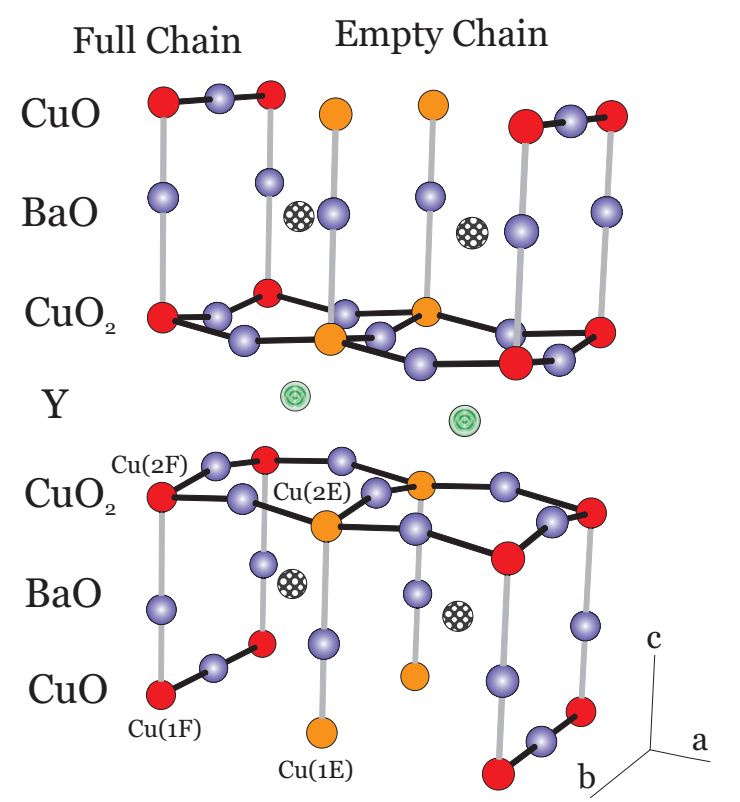

(i) Ortho-II $\left(\mathrm{YBa}_{2} \mathrm{Cu}_{3} \mathrm{O}_{6.5}\right)$

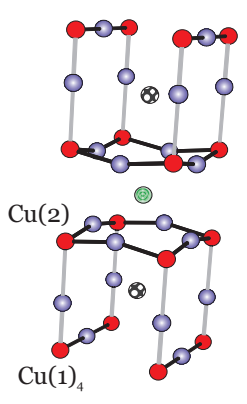

(ii) Ortho-I $\left(\mathrm{YBa}_{2} \mathrm{Cu}_{3} \mathrm{O}_{7}\right)$

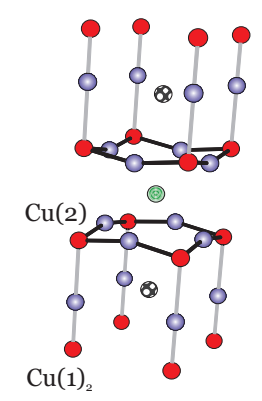

(iii) Tetragonal $\left(\mathrm{YBa}_{2} \mathrm{Cu}_{3} \mathrm{O}_{6}\right)$

\section{$\mathrm{Cu} \odot \quad \mathrm{Ba}$ \\ $\mathrm{O} \odot \mathrm{Y}$ (8)}

FIG. 1: The crystal structure of $\mathrm{YBCO} 6+x$ for (i) $\mathrm{x}=0.5$ (Ortho-II) (ii) $\mathrm{x}=1.0$ (Ortho-I) and (iii) $\mathrm{x}=0.0(\mathrm{~T})$.

contents 19, 20, 21, 22, 23] where either the sample is not stoichiometric or suffers from oxygen inhomogeneity, powder averaging or twinning. Lütgemeier's group 22] was the first to study the effects of oxygen ordering on the NMR properties of YBCO6+x. However, they used aligned powdered samples where the NMR resonances were broadened by non-stoichiometry and oxygen disorder. For Ortho-II ordering only a partially resolved splitting of the resonance line from the $\mathrm{CuO}_{2}$ plane was observed.

With recent enhancements in crystal growth very pure and highly ordered detwinned Ortho-II crystals with large oxygen-ordered domain size have become available 24]. To probe the intrinsic properties of the Ortho-II phase of YBCO we have performed NMR ex- 
periments on highly ordered detwinned Ortho-II single crystals of $\mathrm{YBa}_{2} \mathrm{Cu}_{3} \mathrm{O}_{6.5}$ (YBCO6.5). We report NMR spectra and line assignments for these Ortho-II crystals together with NMR evidence pertaining to the quality of the crystals, specifically regarding the chain lengths and the lack of oxygen disorder. We also report EFG parameters for different $\mathrm{Cu}$ sites in Ortho-II structure along with an analysis regarding lattice and valence charge contributions. We will discuss the relation between oxygen ordering and charge transfer in the Ortho-II structure. It will be shown that these crystals are an extremely important disorder-free prototype of the underdoped state of HTSC.

\section{EXPERIMENT}

Single crystals of YBCO6.5 were grown by a flux method using $\mathrm{BaZrO}_{3}$ crucibles 24]. The crystals were first detwinned and then the oxygen content set to 6.50 during the final annealing process. The NMR properties of two such crystals used in our experiments proved to be identical to within experimental error. Hence we will not distinguish between them. The crystals were $2 \mathrm{~mm} \times 2.5$ $\mathrm{mm}$ wide and $\sim 0.1 \mathrm{~mm}$ thick platelets with the $c$-axis perpendicular to the platelet and $a$ and $b$ axes parallel to the edges of the platelet. The superconducting transitions of the crystals measured by a SQUID magnetometer give $T_{c}=62 \mathrm{~K}$ with a transition width $\Delta T_{c}=0.6 \mathrm{~K}$. $\mathrm{X}$-ray diffraction measurements performed on these crystals show a three-dimensional Ortho-II oxygen ordering with long correlation lengths 148, 430 and $58 \AA$ along the $a, b$ and $c$ crystallographic axes, respectively [24]. The long correlation length along the $b$ (chain) axis indicates the presence of much longer chains than have been available previously [22]. Both the large oxygen-ordered domain size and a small $\Delta T_{c}$ are indicative of high degree of oxygen homogeneity in these crystals. Because twin boundaries promote oxygen clustering, the removal of twin boundaries was found to be essential for obtaining a highly ordered Ortho-II phase 24]. Single crystal X-ray diffraction measurements on our crystals showed at most $10 \%$ of the sample was twinned.

$\mathrm{Cu}$ NMR data were taken between 30 and $250 \mathrm{~K}$ and magnetic fields up to $9 \mathrm{~T}$ with a home built pulsed spectrometer with quadrature detection. The crystal was placed in either a $\mathrm{Pt}$ or $\mathrm{Au}$ rectangular coil tunable from 30 to $100 \mathrm{MHz}$. A rectangular coil was chosen to conform to the shape of the crystal and so maximize the filling factor. The onset of the superconducting transition of the sample was observed in situ at $62 \mathrm{~K}$ by a sharp change in the inductance of the tuned coil at zero applied magnetic field. A powder Al reference sample was located in the coil adjacent to the sample to calibrate the value of the magnetic field at the sample position.

The data were obtained with a standard two-pulse spin-echo sequence, with $4 \mu$ s pulse length and 30 to $40 \mu$ s pulse separation. Copper nuclei have two isotopes
${ }^{63} \mathrm{Cu}$ and ${ }^{65} \mathrm{Cu}$ with spin $\frac{3}{2}$. Each isotope gives one central transition $\left(\frac{1}{2} \leftrightarrow-\frac{1}{2}\right)$ and two satellites $\left( \pm \frac{3}{2} \leftrightarrow\right.$ $\left.\pm \frac{1}{2}\right)$. To obtain the complete $\mathrm{Cu}$ NMR spectrum of the sample we used either field or frequency sweep methods. The spectra were then constructed by superimposing the Fourier transform spectra of the spin echo measured at a certain frequency or field interval. There was no attempt to correct the signal intensity for $T_{2}$ or frequency dependencies in frequency sweep measurements, given the latter's narrow range. Although the samples are high quality, because of their small volume a significant amount of signal averaging was required to obtain a satisfactory signal to noise $(\mathrm{S} / \mathrm{N})$ ratio.

\section{RESULTS AND DISCUSSION}

\section{A. Line Assignment}

Typical ${ }^{63} \mathrm{Cu}$ NMR spectra of central transitions obtained at $60 \mathrm{~K}$ at $75.75 \mathrm{MHz}$ for the applied field parallel to the $a$-axis $\left(\mathbf{H}_{0} \| \mathbf{a}\right)$ are shown in Fig. 2] Similar spectra were also observed for $\mathbf{H}_{0} \| \mathbf{b}$ containing four resonance lines but at different positions. We denote the observed lines in Fig. 2 by A, B, C and D. The spectrum for A-D resonances has also been obtained for ${ }^{65} \mathrm{Cu}$ with intensities consistent with the natural abundances of the two isotopes. These four $\mathrm{Cu}$ resonances are associated with the four inequivalent $\mathrm{Cu}$ sites in the unit cell as expected from the Ortho-II crystal structure (Fig. 11(i)). Two lines $\mathrm{A}$ and $\mathrm{C}$ appear nearly with the same width, integrated area and relaxation rates. Line $\mathrm{B}$ has a very long spinlattice relaxation time (nearly three order of magnitude longer than $\mathrm{A}$ and $\mathrm{C}$ ) and is relatively narrow compared to the rest of the lines. Line $\mathrm{D}$ is broader compared to line $\mathrm{A}$ and $\mathrm{C}$ and has an order of magnitude longer relaxation time. It should be noted that these resonance lines are well resolved and that there appears to be no background signal under these lines. Also note that a relatively long repetition time of $250 \mathrm{msec}$ must be used in order not to saturate line B. Most of the spectra are collected with a shorter repetition time of $25 \mathrm{msec}$ in order to saturate line B which otherwise obscures line A.

Line assignments will be made by comparing the local parameters of the four $\mathrm{Cu}$ sites with those of YBCO6 and YBCO7. To motivate this comparison consider the structural environmemt of each $\mathrm{Cu}$ site. Ortho-II YBCO consists of alternating full and empty chains (see Fig. 11(i)) and hence there are two inequivalent $\mathrm{Cu}$ sites in $\mathrm{CuO}$ chain layers, one in full $\mathrm{Cu}(1 \mathrm{~F})$ and one in empty $\mathrm{Cu}(1 \mathrm{E})$ chains. Correspondingly there are two inequivalent $\mathrm{Cu}$ sites in the $\mathrm{CuO}_{2}$ planes, adjacent to full and empty chains $\mathrm{Cu}(2 \mathrm{~F})$ and $\mathrm{Cu}(2 \mathrm{E})$, respectively. Comparing the crystal structure of Ortho-II with that of YBCO7 (Fig. 11(ii)), one concludes that the local structural environments of $\mathrm{Cu}(2 \mathrm{~F})$ and $\mathrm{Cu}(1 \mathrm{~F})$ are similar to that of $\mathrm{Cu}(2)$ in the plane and four-fold coordinated $\mathrm{Cu}$ in the chain layers $\mathrm{Cu}(1)_{4}$ in YBCO7, respectively. Similarly, 


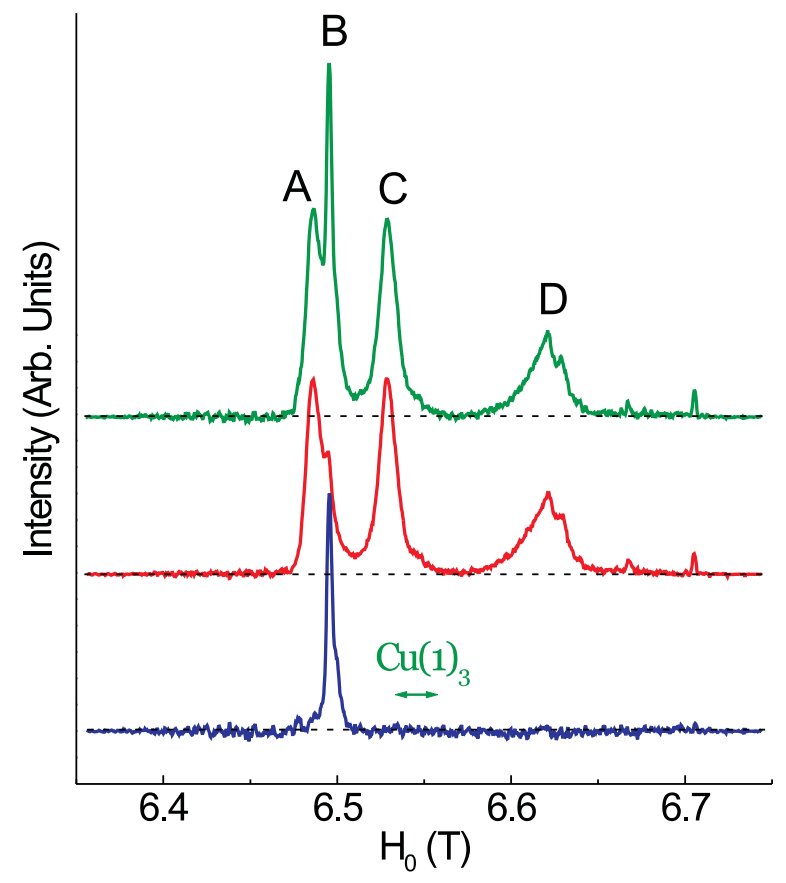

FIG. 2: ${ }^{63} \mathrm{Cu}$ NMR spectra for central transitions at $60 \mathrm{~K}$ and $75.75 \mathrm{MHz}$ for $\mathbf{H}_{0} \| \mathbf{a}$ taken at different repetition time, $\mathrm{T}_{r e p}$ (top panel: $\mathrm{T}_{r e p}=250 \mathrm{msec}$, middle panel: $\mathrm{T}_{r e p}=25 \mathrm{msec}$, and lower panel: the difference). The horizontal arrow indicates the possible range for $\mathrm{Cu}(1)_{3}$. See text for details. The two narrow and small signals in the far right side of the spectra are from $\mathrm{Cu}$ metal in the sample probe.

the $\mathrm{Cu}(2 \mathrm{E})$ and $\mathrm{Cu}(1 \mathrm{E})$ sites have the same local structural environment as $\mathrm{Cu}(2)$ in the plane and two-fold coordinated $\mathrm{Cu}$ in the chain layers $\mathrm{Cu}(1)_{2}$ in YBCO6. NMR parameters for these lines will be compared with the known 25, 26, 27] NMR parameters (Knight shifts, nuclear quadrupole frequency and asymmetry parameters) of the corresponding sites in YBCO7 and YBCO6 to determine the line assignments.

In order to obtain the NMR parameters for the observed lines, we have measured the complete ${ }^{63 / 65} \mathrm{Cu}$ spectrum including the satellite and central transitions at different frequencies and fields along the three crystallographic axes. A typical complete spectrum is shown in Fig. 3 for $\mathbf{H}_{0} \| \mathbf{c}$ at $70 \mathrm{~K}$. Note that for this direction of the applied field, the central and lower satellite transitions of lines $\mathrm{A}$ and $\mathrm{C}$ overlap. The spectra were analyzed using second order perturbation theory for the quadrupolar shift to the Zeeman interaction (see Appendix) for central transitions and by exact diagonalization for $\mathbf{H}_{0}$ parallel to crystallographic axes for the satellites since second order perturbation theory is insufficient for these resonances.

If one writes the resonance frequency $\nu$ (see Appendix) of the central transition to the second order as

$$
\frac{\nu}{\gamma_{n} H_{0}}-1=K_{\text {eff }}=K+\frac{f\left(\nu_{Q}, \eta, \theta, \phi\right)}{1+K} \frac{1}{\left(\gamma_{n} H_{0}\right)^{2}}
$$

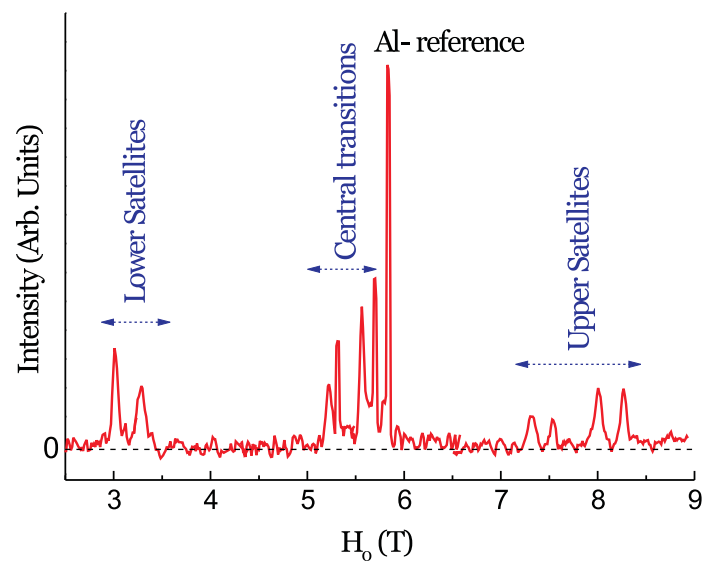

FIG. 3: The complete $\mathrm{Cu}$ NMR spectrum at $70 \mathrm{~K}$ and 64.85 $\mathrm{MHz}$ for $\mathbf{H}_{0} \| \mathbf{c}$. For this direction, the central transitions and lower satellites of A and C lines overlap.

it is easily seen that $K_{\text {eff }}$ is a linear function of $\frac{1}{\left(\gamma_{n} H_{0}\right)^{2}}$ with the intercept equal to $K$ and the slope proportional to the quadrupolar coupling constant for that direction. For example, in Fig. [4 we have shown this data for the central transition of the line D obtained at $70 \mathrm{~K}$ and for $\mathbf{H}_{0} \| \mathbf{b}$. We have used the position of the center of gravity of this line to obtain $K_{\text {eff }}$ since this line is broad at low temperatures. The Knight shifts and EFG parameters are obtained for all sites with a simultaneous fit of the central transition and satellite data in the three field orientations [28].

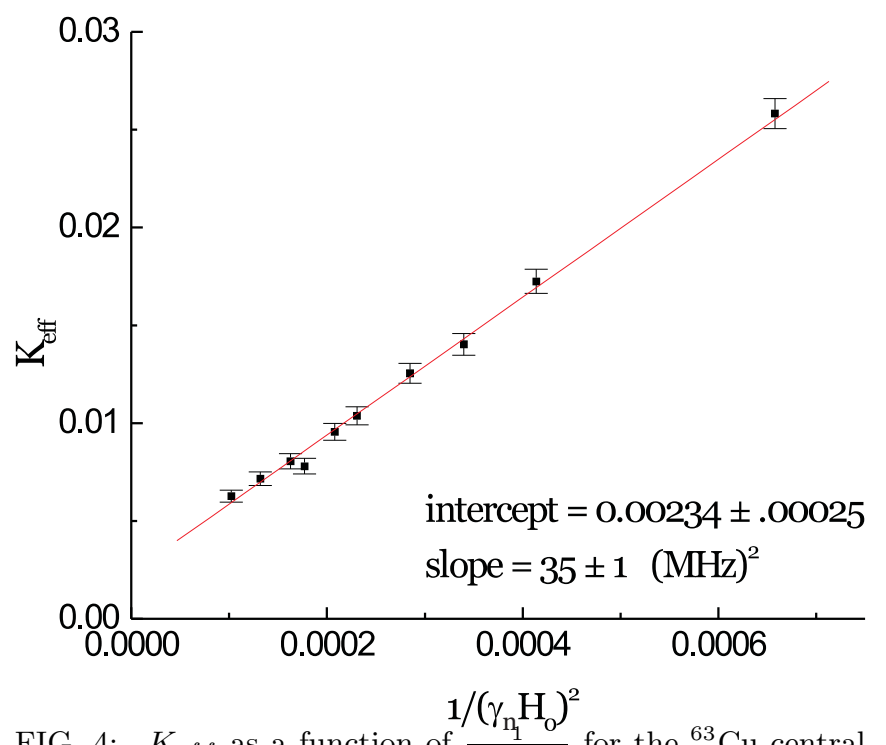

FIG. 4: $\quad K_{\text {eff }}$ as a function of $\frac{\left(_{n_{1}} \mathbf{H}^{2}\right.}{\left(\gamma_{n} \mathbf{H}_{0}\right)^{2}}$ for the ${ }^{63} \mathrm{Cu}$ central transition of the D line at $70 \mathrm{~K}, \mathbf{H}_{0} \| \mathbf{b}$.

The NMR parameters for the four observed lines in the normal state are reported in Table【 For comparison we have listed the NMR parameters of $\mathrm{Cu}(2)$ and $\mathrm{Cu}(1)_{4}$ 
sites in $\mathrm{YBCO} 7$ and $\mathrm{Cu}(2)$ and $\mathrm{Cu}(1)_{2}$ sites in YBCO6 in Table 25, 26, 27]. We can readily assign the four resonances observed in Ortho-II to the four different crystallographic sites by comparing their NMR parameters to those of $\mathrm{Cu}$ sites in YBCO7 and YBCO6. This comparison shows that the two lines $\mathrm{A}$ and $\mathrm{C}$, with very similar features, come from the planes; A from the planar $\mathrm{Cu}$ sites adjacent to the full chains $\mathrm{Cu}(2 \mathrm{~F})$ and $\mathrm{C}$ from the planar $\mathrm{Cu}$ sites adjacent to the empty chains $\mathrm{Cu}(2 \mathrm{E})$. The EFG principal axis for both sites is along the $c$ axis which is the same for the $\mathrm{Cu}(2)$ sites in YBCO7 and YBCO6. The asymmetry parameter for these sites is very small (nearly axially symmetric) as with the $\mathrm{Cu}(2)$ sites in YBCO7 and YBCO6. The slightly larger asymmetry parameter observed in Ortho-II might be explained by crystal structure arguments since Ortho-II structure has a lower symmetry than the Ortho-I or tetragonal phases. The $\mathrm{Cu}(2 \mathrm{~F})$ and $\mathrm{Cu}(2 \mathrm{E})$ resonances appear at different positions because of their different quadrupolar coupling constants. Since the quadrupolar coupling depends on the ion's surrounding charge distribution as well as the local charge at the position of the ion, a different coupling is expected for the two planar $\mathrm{Cu}$ sites as observed. We will discuss the contribution of the lattice and valence electrons to this coupling in more detail below. The $\nu_{Q}$ for $\mathrm{Cu}(2 \mathrm{E})$ is higher than the observed one in YBCO6, consistent with the trend of $\nu_{Q}$ vs. doping 29]. The same intensity of the $\mathrm{Cu}(2 \mathrm{~F})$ and $\mathrm{Cu}(2 \mathrm{E})$ lines 30 within our $\mathrm{S} / \mathrm{N}$ is an indication that there are equal numbers of these sites in the crystal. Hence there is a high degree of ordering in the chain layers with a periodic sequence of full and empty chains. The mere fact that we have observed only two distinct $\mathrm{Cu}(2)$ signals for both satellite and central transitions (Fig. 3) is suggestive of a complete ordering of the full and empty chains. In a disordered structure the signal would merge to one broad resonance with some splitting depending on the degree of ordering. Such partially resolved splittings for the planar $\mathrm{Cu}$ resonances is typical 22] in previous generations of $\mathrm{YBCO} 6+x$ samples.

The resonance line B appears in the same frequency range as $\mathrm{Cu}(2 \mathrm{~F})$ but can be distinguished by its much longer relaxation time. In fact, among the four observed resonance lines, B has the smallest width, Knight shift and the longest relaxation time (of order seconds) comparable with that of $\mathrm{Cu}(1)_{2}$ in the insulating tetragonal phase of YBCO6 31]. A comparison of the NMR parameters of this line with $\mathrm{Cu}(1)_{2}$ present in YBCO6 with empty chains leads us to assign this line to $\mathrm{Cu}(1 \mathrm{E})$ in the Ortho-II structure. Similar to $\mathrm{Cu}(1)_{2}$, the $\mathrm{Cu}(1 \mathrm{E})$ resonance has axial symmetry. The long spin-lattice relaxation time is interpreted as a result of a low conduction electron density at the resonant nucleus. This is consistent with the $\mathrm{Cu}$ ions being in the $3 \mathrm{~d}^{10}$ electronic configuration. A small Knight shift is also consistent with a low density of conduction electrons at this site. In addition, the fact that the resonance signal from this site is very narrow is in agreement with this conclusion because insulating sites tend to be much more uniform in nature than conducting ones as they lack an inhomogeneous $K_{s}$ component. The $\mathrm{Cu}(1 \mathrm{E})$ linewidth is $40 \mathrm{kHz}$ at $60 \mathrm{~K}$ with $\mathbf{H}_{0} \| \mathbf{a}=6.5 \mathrm{~T}$ for different directions of applied field and becomes even narrower at higher temperatures.

Line $\mathrm{D}$ is broader than other resonances, at least at low temperatures. We have found a large value for its asymmetry parameter (see Table I). The highly asymmetric behavior of the EFG is reminiscent of $\mathrm{Cu}(1)_{4}$ in YBCO7. This fact together with its similar $\nu_{Q}$ has led us to assign this line to the $\mathrm{Cu}(1 \mathrm{~F})$ site. An examination of $\mathrm{Cu}(1 \mathrm{~F})$ site symmetry explains its highly asymmetric nature. In the Ortho-II structure, the $\mathrm{Cu}(1 \mathrm{~F})$ sites are surrounded by four oxygen ions, two chain oxygens and two apical oxygens. Since these oxygen neighbors are not all equivalent, a non-symmetric EFG is expected. To understand the nature of the broadening of this line at low temperatures, we have studied the line shape and relaxation rates for several directions of the applied field. We attribute this broadening to the presence of Friedel-like oscillations nucleated at the chain ends. The details of this study will be presented elsewhere 32 .

We have confirmed that the ratio of quadrupolar shifts of the two isotopes measured at the same site differs by their quadrupole moment $\left({ }^{63} \mathrm{Q} /{ }^{65} \mathrm{Q}=1.079[28]\right.$ ) for all sites. This indicates that no internal magnetic field, due to static magnetic moments, is present at these $\mathrm{Cu}$ sites since such a field would shift or broaden the lines. This is in disagreement with some neutron-scattering measurements where a moment of $\sim 0.05 \mu_{B}$ is observed 33] in YBCO6.5 and of $\sim 0.02 \mu_{B}$ in YBCO6.6 34 for copper sites in the $\mathrm{CuO}_{2}$ planes, and with $\mu$ SR results on YBCO6.67 showing 35] the existence of static magnetism in these planes. A magnetic moment of the order of $\sim$ $0.02 \mu_{B}$ would have shifted or broadened the $\mathrm{Cu}(2 \mathrm{~F} / \mathrm{E})$ lines by about $3 \mathrm{MHz}$ which we would have easily observed. More recent neutron scattering experiments on Ortho-II found 36] no evidence for the presence of static magnetic moments. Also, in a further work, it was shown 37 that the $\mu \mathrm{SR}$ results might arise from charge inhomogeneity rather than magnetic order.

To further elucidate this apparent discrepency several factors should be noted. As reported by Sidis et al. 33 their static moments fluctuate on a nanosecond time scale which would average out these moments on the NMR time scale. However this still does not settle the discrepancy with the work of Stock et al. [36]. In this experiment the Ortho-II sample is much more stoichiometric than that of Sidis et al.or the YBCO6.6 sample of Mook et al.. Thus, as suggested by Stock et al., oxygen disorder in the chains may be responsible for establishing magnetic order there. With respect to the $\mu \mathrm{SR}$ results, Sonier et al. [37] have suggested that for samples with $x>$ 6.5 local charge inhomogeneity, caused by CDW in the chains as shown by Grévin et al. 8] could lead to magnetic moments via the stripe mechanism. However for the Ortho-II concentration of $x=6.5$ no $\mu \mathrm{SR}$ signature of a CDW was detected. From our work on the $\mathrm{Cu}(1 \mathrm{~F})$ line mentioned above 32$]$ we have established that a Friedel- 
TABLE I: Experimental NMR parameters for the four observed lines at $70 \mathrm{~K}$. Knight shifts are reported in units of percent and $\nu_{Q}$ in MHz. EFG-PA represents the direction of $V_{z z}$. Site assignment is based on the comparison of these parameters with those of $\mathrm{Cu}$ sites in YBCO7 and YBCO6. For details see the text.

\begin{tabular}{llcccccc}
\hline \hline resonance & site & $\mathrm{K}_{a}$ & $\mathrm{~K}_{b}$ & $\mathrm{~K}_{c}$ & $\nu_{Q}$ & \multicolumn{2}{c}{\begin{tabular}{c} 
EFG-PA \\
\hline $\mathrm{A}$
\end{tabular} $\mathrm{Cu}(2 \mathrm{~F})_{\mathrm{B}}$} \\
$\mathrm{Cu}(1 \mathrm{E})$ & $-0.25 \pm 0.01$ & $0.25 \pm 0.01$ & $1.18 \pm 0.01$ & $30.55 \pm 0.03$ & $0.018 \pm 0.002$ & $\mathrm{c}$ \\
$\mathrm{C}$ & $\mathrm{Cu}(2 \mathrm{E})$ & $0.26 \pm 0.01$ & $0.24 \pm 0.01$ & $1.14 \pm 0.01$ & $27.43 \pm 0.03$ & $0.022 \pm 0.002$ & $\mathrm{c}$ \\
$\mathrm{D}$ & $\mathrm{Cu}(1 \mathrm{~F})$ & $0.9 \pm 0.1$ & $0.23 \pm 0.03$ & $0.23 \pm 0.05$ & $19.6 \pm 0.1$ & $0.8 \pm 0.1$ & $\mathrm{c}$ \\
\hline \hline
\end{tabular}

TABLE II: NMR parameters for $\mathrm{Cu}(2)$ and $\mathrm{Cu}(1)_{4}$ sites in $\mathrm{YBCO} 7$ and $\mathrm{Cu}(2)$ and $\mathrm{Cu}(1)_{2}$ sites in YBCO6 materials. Knight shifts are in units of percent, $\nu_{Q}$ in MHz. EFG-PA represents the direction of $V_{z z}$.

\begin{tabular}{llcccccc}
\hline \hline & site & $\mathrm{K}_{a}$ & $\mathrm{~K}_{b}$ & $\mathrm{~K}_{c}$ & $\nu_{Q}$ & $\eta$ \\
\hline $\mathrm{YBCO}^{a}$ & $\mathrm{Cu}(2)$ & $0.59 \pm 0.04$ & $0.59 \pm 0.04$ & $1.267 \pm 0.001$ & $31.54 \pm 0.05$ & $0.01 \pm 0.01$ & $\mathrm{EFG}-\mathrm{PA}$ \\
& $\mathrm{Cu}(1)_{4}$ & $1.38 \pm 0.07$ & $0.55 \pm 0.07$ & $0.60 \pm 0.04$ & $19.35 \pm 0.08$ & $0.95 \pm 0.02$ & $\mathrm{a}$ \\
\hline $\mathrm{YBCO}^{b}$ & $\mathrm{Cu}(2)$ & $0.47 \pm 0.02$ & & $1.335 \pm 0.005$ & $23.80 \pm 0.2$ & 0 & $\mathrm{c}$ \\
& $\mathrm{Cu}(1)_{2}$ & $0.18 \pm 0.01$ & & $-0.117 \pm 0.003$ & $29.54 \pm 0.02$ & 0 & $\mathrm{c}$ \\
\hline \hline
\end{tabular}

${ }^{a}$ The Knight shifts are from Ref.[25], $\nu_{Q}$ and $\eta$ are taken from Ref. [26] all at $100 \mathrm{~K} .{ }^{b}$ Taken from Ref.[27] at 505 K.

like spin-density oscillation exists in the chains of sufficient magnitude to produce the field, of order 1 gauss, necessary to account for the Ortho-II $\mu$ SR results. Thus the $\mathrm{CDW} /$ stripe mechanism need not be invoked in the case of stoichiometric Ortho-II YBCO.

\section{B. Chain Length}

We now estimate the chain lengths from our NMR data. In YBCO6+x compounds with intermediate oxygen content, a resonance line with $\nu_{N Q R} \simeq 23 \mathrm{MHz}$ is observed 22] and is associated with three-fold coordinated $\mathrm{Cu}(1)_{3}$ sites corresponding to the terminal $\mathrm{Cu}$ ion of the full chains with a relative abundance that diminishes with increasing length of the chain segments. A similar value of $\nu_{Q}$ for $\mathrm{Cu}(1)_{3}$ and $\mathrm{Cu}(1 \mathrm{~F})$ sites together with the fact that $\mathrm{Cu}(1 \mathrm{~F})$ is very broad at low temperatures, makes it difficult to obtain a precise value for the concentration of these sites in our sample. As discussed above we expect the concentration of $\mathrm{Cu}(1)_{3}$ to be low. In fact, in the complete NMR spectrum for Ortho-II measured between 1 to $9 \mathrm{~T}$, to within our $\mathrm{S} / \mathrm{N}$ we did not observe any signal that could not be assigned to the four inequivalent sites in the structure. Hence, we can only estimate an upper limit on the number of $\mathrm{Cu}(1)_{3}$ sites from our $\mathrm{S} / \mathrm{N}$ and ultimately a lower limit on the chain length in our sample.

To put a quantitative limit on the concentration of $\mathrm{Cu}(1)_{3}$ sites, we consider two sets of spectra. The first one was taken at $250 \mathrm{~K}$ where $\mathrm{Cu}(1 \mathrm{~F})$ is considerably narrower than the one at $70 \mathrm{~K}$. The Friedel-like oscillations are thermally smeared out at this high temperature. In Fig. [5 we have shown this spectra for $\mathbf{H}_{0} \| \mathbf{c}$. We have used the established NMR parameters of the $\mathrm{Cu}(1)_{3}$ sites [22, 38] $\left(\nu_{N Q R}=23.77 \mathrm{MHz}, \eta=0.3\right.$ and $\left.V_{z z} \| \mathbf{b}\right)$ to calculate its possible position. The horizontal arrow in Fig. 5 covers the range consistent with parameter uncertainties. From the relative intensities of $\mathrm{Cu}(1 \mathrm{~F}), I_{4}$, and $\mathrm{Cu}(1)_{3}, I_{3}$, one is able in principle to find the mean number of oxygen atoms in the full chains (chain length) according to the relation 22 ]: $\mathrm{n}=1+2 I_{4} / I_{3}$. Since we have not observed any additional peaks due to $\mathrm{Cu}(1)_{3}$, we can only put a limit on the maximum intensity of $I_{3}$ from our $\mathrm{S} / \mathrm{N}$. To obtain this limit, we have assumed the $\mathrm{Cu}(1)_{3}$ line has the same NQR width as observed by Yasuoka et al. 20] leading to an NMR linewidth of $66 \mathrm{kHz}$. If we assume we can detect such a line with $\mathrm{S} / \mathrm{N}$ of two then we obtain a lower limit of 32 lattice constants for the chain length. The inset to Fig. [ 5 illustrates the $\mathrm{Cu}(1)_{3}$ signal expected for $n=30$ and $n=60$.

The second set of spectra was taken at $60 \mathrm{~K}$ with $\mathbf{H}_{0} \| \mathbf{a}$. In this case we use the fact that the relaxation rate of the $\mathrm{Cu}(1)_{3}$ site is relatively slow 39 to extract only the slowly relaxing components. As illustrated in Fig. 2 two spectra taken at a repetition time of $25 \mathrm{msec}$. and 250 msec. have been subtracted to reveal the $\mathrm{Cu}(1 \mathrm{E})$ line as expected and no other line. As above we put an upper limit on the chain length given our S/N. The calculated range for $\mathrm{Cu}(1)_{3}$ is displayed in Fig. 22 Our estimate of the chain length from this analysis is 50 lattice constants.

These estimates are consistent with the XRD result of 110 lattice constants for the domain size along the chain direction. Using the ASYNNNI model we have performed Monte Carlo calculations to determine what the domain interface looks like for the two cases of (a) the case of a twin boundary and (b) parallel chains that are out of phase, i.e. FEFE adjoining EFEF (see Fig. 6). In both cases a linear boundary is preferred as the ground state with $\mathrm{Cu}(1)_{3}$ atoms at the domain boundary. Thus 
we expect to have three-fold coordinated copper sites at least along the domain boundaries normal to $\mathbf{b}$ and more along the twin boundaries to the extent that they exist in our crystals. Hence the relative concentration of $\mathrm{Cu}(1)_{3}$ sites should be $I_{3} / I_{4} \simeq 2 \%$ from the XRD results whereas our detection limit for $I_{3} / I_{4} \simeq 4 \%$. From the two values for $\mathrm{n}$ obtained above, the consistent estimate for the lower limit of the chain lengths is 50 lattice constants.

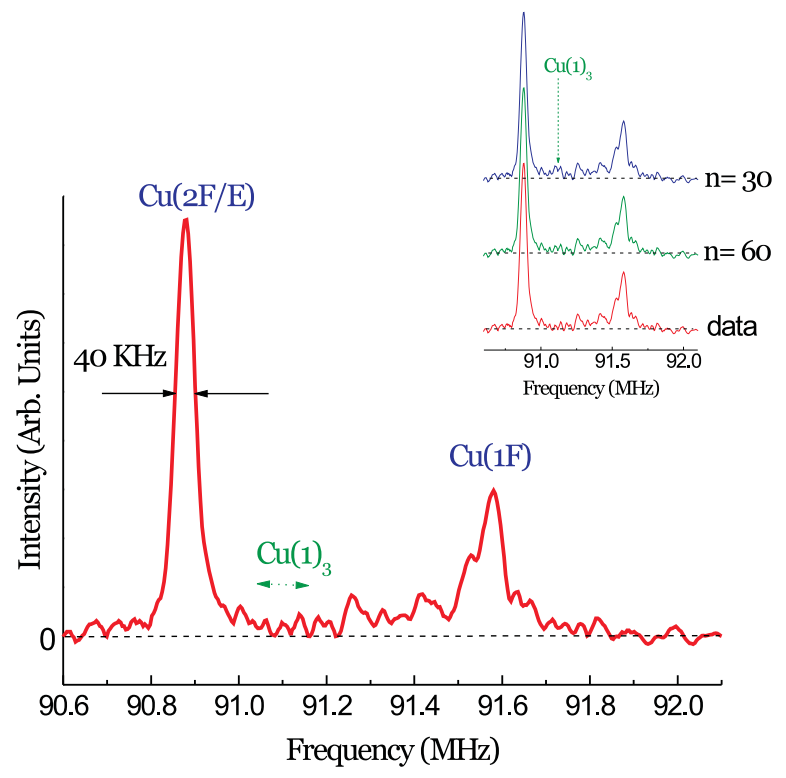

FIG. 5: ${ }^{63} \mathrm{Cu}$ NMR spectrum at $250 \mathrm{~K}$ and $\mathbf{H}_{0}(=7.96 T) \| \mathbf{c}$. The arrow shows the estimated range of the $\mathrm{Cu}(1)_{3}$ resonance. Inset illustrates presence of $\mathrm{Cu}(1)_{3}$ line synthetically added to the data for two chain lengths. This line is barely visible for $\mathrm{n}=30$ and undetectable for $\mathrm{n}=60$.

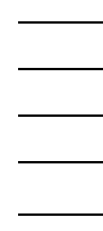

(a)

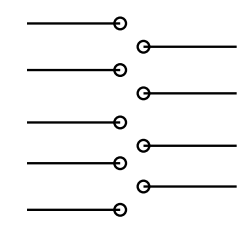

(b)
FIG. 6: Domain interfaces calculated by Monte Carlo calculations: (a) twin boundary and (b) parallel chains out of phase. Line segments represent $\mathrm{Cu}-\mathrm{O}-\mathrm{Cu}$ chains. Chains are terminated with $\mathrm{Cu}(1)_{3}$ sites denoted with circles.

In NMR studies on HTSCs, large linewidths have been an obstacle to investigating intrinsic properties. The problem arises from the fact it is not readily obvious whether these large linewidths arise from imperfections of the structure such as oxygen disorder or are an intrinsic property of these materials. However, recent im- provements in sample preparation techniques have resulted in the observation of narrower lines [0] emphasizing the importance of sample quality in determining the properties of HTSC materials. In general, the observed linewidths can be attributed to the distributions in either the EFG parameters (arising from the structure) and/or the Knight shift. Our analysis of the linewidths of the central transitions vs. applied magnetic field as well as a comparison between the linewidths of central and satellite transitions indicates that the line broadening for $\mathrm{Cu}(2 \mathrm{~F} / \mathrm{E})$ and $\mathrm{Cu}(1 \mathrm{E})$ sites is mainly caused by a distribution of EFG parameters while for $\mathrm{Cu}(1 \mathrm{~F})$ it is mainly due to a distribution of the Knight shift. The linewidths of all resonance lines decreases with increasing temperature. The planar $\mathrm{Cu}$ sites for $\mathbf{H}_{0} \| \mathbf{c}$ have a linewidth of $40 \mathrm{kHz}$ at $250 \mathrm{~K}$. To our knowledge these NMR linewidths are the smallest observed for YBCO6.5 samples [40], another indication of high sample quality.

Oxygen disorder is a feature of non-stoichiometric samples. Erb et al. [40] have compared the NMR spectra of YBCO7 and YBCO6.9. The former has very narrow NMR lines. For example the linewidth of $\mathrm{Cu}(1)_{4}$ is 22 $\mathrm{kHz}$ with $\mathbf{H}_{0}=9 \mathrm{~T} \| \mathbf{c}$ at room temperature. When the oxygen concentration is reduced by 0.1 the linewidth increases to $183 \mathrm{kHz}$, as a result of oxygen disorder. Also noted was the presence of $\mathrm{Cu}(1)_{2}$ with a linewidth of $13 \mathrm{kHz}$. They argue that this narrow linewidth is a result of oxygen clustering which provides a more uniform environment for the $\mathrm{Cu}(1)_{2}$ sites than would be the case for randomly distributed oxygen defects. They also argue that the absence of a $\mathrm{Cu}(1)_{3}$ signal is consistent with oxygen clustering as this three-fold coordinated site forms the boundary between full and empty chains. As our linewidths are similar to that of YBCO7 we can infer that there are few oxygen defects in our Ortho-II YBCO6.5 crystals. We find that our $\mathrm{Cu}(1 \mathrm{E})$ linewidth for fields along $\mathbf{a}$ and $\mathbf{b}$ is $30 \mathrm{kHz}$ at $200 \mathrm{~K}$ consistent with few oxygen defects. Our narrow $\mathrm{Cu}(1 \mathrm{~F})$ line at these high temperatures is also consistent with few oxygen defects. This is relevant to the $\mathrm{Cu}(1)_{3}$ concentration. Although we expect no substantial number of oxygen defects in stoichiometric YBCO6.5 the absence of a $\mathrm{Cu}(1)_{3}$ line indicates that our crystals are indeed substantially stoichiometric.

From the NMR spectrum obtained for $\mathbf{H}_{0} \| \mathbf{a}$ and $\mathbf{b}$, we are also able to investigate the degree of twinning in our crystal. Fig. 7 shows the ${ }^{63} \mathrm{Cu}$ NMR spectrum for $\mathrm{Cu}(2 \mathrm{~F})$ and $\mathrm{Cu}(2 \mathrm{E})$ at $60 \mathrm{~K}, 75.75 \mathrm{MHz}$. A remnant of $\mathrm{Cu}(1 \mathrm{E})$ is observed on the high side of $\mathrm{Cu}(2 \mathrm{~F})$ as our pulse sequence to saturate the $\mathrm{Cu}(1 \mathrm{E})$ signal is not $100 \%$ efficient. It is evident from the lack of asymmetry of the two lines that there is no obvious indication of the presence of twinning in our crystal. Quantitatively we find by adding an admixture of the twinned spectrum that to within our $\mathrm{S} / \mathrm{N}$, there could be a maximum of ten percent twinning present in our sample. This is consistent with our XRD measurements discussed above. 


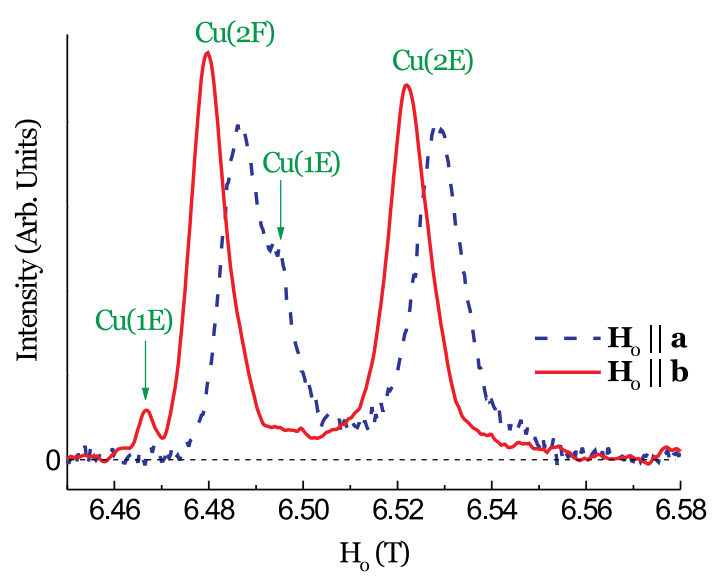

FIG. 7: ${ }^{63} \mathrm{Cu}$ NMR spectrum at $60 \mathrm{~K}$ and $75.75 \mathrm{MHz}$ for $\mathbf{H}_{0} \| \mathbf{a}$ and $\mathbf{b}$. The spectra are normalized to the integrated intensity of $\mathrm{Cu}(2 \mathrm{E})$ line. Note that the feature on the right side of $\mathrm{Cu}(2 \mathrm{~F})$ for $\mathbf{H}_{0} \| \mathbf{a}$ is due to the imperfect subtraction of $\mathrm{Cu}(1 \mathrm{E})$ signal. This of course does not affect the $\mathrm{Cu}(2 \mathrm{E})$ lineshape.

\section{Charge Transfer and Oxygen Ordering}

Oxygen ordering in the chain layer may have an effect on charge transfer to the planes. We now investigate this possibility through the planar copper EFG values. Considering the intinerant holes in the $\mathrm{CuO}_{2}$ planes, $\nu_{Q}$ for the two sites $\mathrm{Cu}(2 \mathrm{E})$ and $\mathrm{Cu}(2 \mathrm{~F})$ in these planes might be expected to be the same. However, we have observed a splitting of the $\mathrm{Cu}(2)$ resonance. The coupling of the nuclei's quadrupole moment with its environment determines $\nu_{Q}$ which is directly proportional to the electric field gradient at the nucleus. The local EFGs reflect the structural and crystallographic aspects of the compound and provide information about ionicity and bonding. The EFG at the nuclear site is determined by two contributions: one arising from the charges on the neighboring ions (lattice contribution) $V_{z z}^{\text {lat }}$ and the second arising from the incomplete electronic shells (valence contribution) $V_{z z}^{v a l}$ of the ion under consideration. The total EFG may be written as 41.

$$
V_{z z}=\left(1-\gamma_{\infty}\right) V_{z z}^{l a t}+(1-R) V_{z z}^{v a l}
$$

where $\gamma_{\infty}$ and $R$ are the anitishielding factors which account for the antishielding effect of the core electrons of the central ion on the lattice and valence contributions, respectively. From this equation, if one calculates the lattice contribution, one can obtain the valence contribution from the data. To what extent does the $\mathrm{Cu}(2)$ splitting stem from chain ordering in the Ortho-II structure (lattice contribution) or from differing charge transfer (valence contribution)? To address this question, we have used the point charge model (PCM) to calculate the
TABLE III: Calculated lattice EFG values, $\mathrm{V}^{\text {lat }}$ in units of $\mathrm{MHz}$, based on the point charge model.

\begin{tabular}{ccccccc}
\hline \hline site & $\mathrm{V}_{a a}^{\text {lat }}$ & $\mathrm{V}_{b b}^{\text {lat }}$ & $\mathrm{V}_{c c}^{\text {lat }}$ & $\eta^{\text {lat }}$ & $\mathrm{EFG}-\mathrm{PA}$ & $(1-\mathrm{R}) \mathrm{V}_{z z}^{\text {val }}$ \\
\hline $\mathrm{Cu}(2 \mathrm{~F})$ & -1.140 & -1.094 & 2.232 & 0.020 & $\mathrm{c}$ & -71.62 \\
$\mathrm{Cu}(2 \mathrm{E})$ & -1.204 & -1.078 & 2.282 & 0.055 & $\mathrm{c}$ & -69.43 \\
$\mathrm{Cu}(1 \mathrm{~F})$ & 4.182 & -1.791 & -2.391 & 0.143 & $\mathrm{a}$ & -96.55 \\
$\mathrm{Cu}(1 \mathrm{E})$ & 2.338 & 2.476 & -4.814 & 0.029 & $\mathrm{c}$ & -2.25 \\
\hline \hline
\end{tabular}

lattice contributions to the observed EFG values for the four $\mathrm{Cu}$ sites in Ortho-II structure. Although the PCM is simplistic we are interested only in relative differences and not the absolute values of EFG parameters.

Applying the formal valence description to Ortho-II YBCO results in $\mathrm{Y}^{+3} \mathrm{Ba}_{2}^{+2} \mathrm{Cu}_{3}^{+2} \mathrm{O}_{6.5}^{-2}$ ionic configuration. However this neglects the fact that $\mathrm{Cu}(1 \mathrm{E})$ is in the $3 \mathrm{~d}^{10}$ state with charge +1 . The resulting extra hole is taken to be distributed over the $\mathrm{CuO}_{2}$ planes. We have taken the planar $\mathrm{Cu}$ sites to have a valence of +2.083 , the planar $\mathrm{O}$ sites a valence of -1.917 , the $\mathrm{Cu}$ sites in the full chain a valence of +2 , the $\mathrm{Cu}$ in the empty chain a valence of +1 , the rest of the oxygens a valence of -2 and $\mathrm{Ba}$ and $\mathrm{Y}$ in +2 and +3 , respectively. We have used the atomic positions obtained [42] by single crystal X-ray diffraction measurements at $80 \mathrm{~K}$. It should be noticed that due to the alternating nature of the full and empty chains, some atomic displacements are observed 42] from the mean position of atoms, most notably Ba. This is attributed to the lattice tendency to minimize its electrostatic energy 433. We have summed to convergence over the contributions within a crystal containing $\pm 50, \pm 100$ and \pm 30 unit cells along the $a, b$, and $c$-axes, respectively. The resulting values for the principal components of EFG for all sites are given in Table III The (1-R) $\mathrm{V}_{z z}^{v a l}$ values are obtained using eq. 3.2 the experimental values of $\nu_{Q}$ and $\gamma_{\infty}$ [4] for $\mathrm{Cu}^{+2}=-17.4$ and $\mathrm{Cu}^{+1}=-5.2$ in a crystalline environment.

The calculated values produce the correct EFG principal axes. Furthermore, small values of the asymmetry parameters for $\mathrm{Cu}(2 \mathrm{~F} / \mathrm{E})$ indicate a small deviation of these sites from tetragonal symmetry in accordance with our experimental results. The directions of the principal axes and the values of $\eta$ are not expected to be affected so much by the electronic structure of the subject ion since this effect is isotropic to first order. This explains the success of the point charge model in predicting the principal axes and $\eta$ values. A larger value of $\eta^{\text {lat }}$ is obtained for the $\mathrm{Cu}(1 \mathrm{~F})$ site. This value is not as large as the observed one since it does not contain the asymmetric ligand contribution.

Since the $\mathrm{Cu}(2 \mathrm{~F} / \mathrm{E})$ and $\mathrm{Cu}(1 \mathrm{~F})$ sites have +2 valence, one would expect 45] a valence contribution, (1$\mathrm{R}) \mathrm{V}_{z z}^{v a l} \approx-70 \mathrm{MHz}$, to the EFG from a hole in the $3 \mathrm{~d}^{9}$ state and a zero contribution for $\mathrm{Cu}(1 \mathrm{E})$ sites in the $3 \mathrm{~d}^{10}$ state. Our values obtained for this contribution to the EFG for $\mathrm{Cu}(1 \mathrm{E})$ site is small and supports this charge 
configuration for this site. We find a difference in valence contribution of $2.19 \mathrm{MHz}$ between the $\mathrm{Cu}(2 \mathrm{~F})$ and $\mathrm{Cu}(2 \mathrm{E})$ sites. Comparing this value to the experimentally observed $3.15 \mathrm{MHz}$, we conclude that the valence (local moment) contribution to the EFG difference at these sites is dominant. This can be understood as resulting from a different charge transfer for these two sites; the full chains act as the charge reservoir whereas the empty chains do not. Charge transfered from the full chains resides at both the $\mathrm{Cu}(2 \mathrm{~F})$ and $\mathrm{Cu}(2 \mathrm{E})$ sites but with a larger amount at $\mathrm{Cu}(2 \mathrm{~F})$, the site closer to the full chain. The inadequacy of the point charge model is apparent for the $\mathrm{Cu}(1 \mathrm{~F})$ site not only in its prediction of the asymmetry parameter but also in the valence contribution [46]. In this case asymmetric ligand effects are mostly responsible.

To investigate the effects of the domain walls on the EFG values, we have introduced different types of domain walls and then calculated the EFG parameters for different sites. This calculation shows that the domain boundaries affect the $\mathrm{Cu}(1 \mathrm{~F} / \mathrm{E})$ sites the most and the $\mathrm{Cu}(2 \mathrm{~F} / \mathrm{E})$ sites the least. Our results also show that the bulk values of the lattice contribution to $\nu_{Q}$ are essentially recovered just one lattice constant away from the domain walls.

\section{CONCLUSIONS}

Line assignments for YBCO6.5 have been made. The lack of an observable NMR signal from the chain ends at the domain walls confirms the long chain lengths suggested by X-ray diffraction. We place a lower limit on the chain length of 50 lattice constants. Consistent with this is the narrow linewidths comparable to those of the best YBCO7 crystals. This is a result of little oxygen disorder in these crystals.

Thus these crystals are a much better realization of

the stoichiometric YBCO6.5 than previously available. Knight shift and spin-lattice relaxation measurements are in progress to investigate the pseudogap. In addition, as mentioned above, the long chains appear to support Friedel-like oscillations nucleated at the chain ends 32].

Another consequence of oxygen ordering in the chain layer is the effect it has on the $\mathrm{CuO}_{2}$ planes via charge transfer. This is manifested in the $\mathrm{Cu}(2 \mathrm{E} / \mathrm{F})$ splitting. Our analysis concludes that most of this splitting is due to differential charge transfer from the chains.

Finally, previous reports of static moments in YBCO6.5 are inconsistent with our results.

\section{Acknowledgments}

We would like to thank J.P. Castellane and B. Gaulin for performing the single crystal $\mathrm{X}$-ray diffraction measurements on our crystals. This work was supported by the NSERC of Canada.

\section{APPENDIX}

A straightforward calculation 47] to second order reveals the transitions frequencies for $\operatorname{spin} \mathrm{I}=\frac{3}{2}$ :

$$
\begin{gathered}
\nu=\nu^{(0)}+\nu^{(1)}+\nu^{(2)} \\
\nu^{(0)}=\gamma_{n} H_{0}(1+K) \\
\nu^{(1)}=\frac{1}{2} \nu_{Q}\left(m-\frac{1}{2}\right)\left[3 \cos ^{2} \theta-1-\eta \sin ^{2} \theta \cos 2 \phi\right]
\end{gathered}
$$

where $K$ is the Knight shift obtained by

$$
K=K_{x} \sin ^{2} \theta \cos ^{2} \phi+K_{y} \sin ^{2} \theta \sin ^{2} \phi+K_{z} \cos ^{2} \theta
$$

and $\theta$ and $\phi$ are the polar angles of the applied magnetic field with respect to the principal axis frame which is assumed to be the same for both the Knight shift tensor and EFG tensor. $\gamma_{n}$ is the nuclear gyromagnetic ratio and $H_{0}$ is the applied magnetic filed. $\eta$ is the asymmetry parameter, defined as $\eta=\frac{V_{x x}-V_{y y}}{V_{z z}}$, where $\left|V_{x x}\right| \leq\left|V_{y y}\right| \leq$ 
$\left|V_{z z}\right|$ are the principal components of the electric field gradient (EFG) tensor at the $\mathrm{Cu}$ site and $\nu_{Q}=e Q V_{z z} / h$, $e$ the elementary electric charge, and $Q$ is the electric quadrupole moment of the nucleus under study. The central and satellite transition resonance frequencies are obtained for $m=\frac{1}{2}$ and $m=\frac{3}{2},-\frac{1}{2}$, respectively.
[†] Current address: Department of Chemistry, University of British Columbia.

[1] J.G. Bednorz and K.A. Muller, Z. Phys. B 64 (1986) 189.

[2] M.K. Wu, J.R. Ashburn, and C.J. Torng et al. Phys. Rev. Lett. 58 (1987) 908.

[3] For an experimental survey on pseudogap phenomena refer to: Tom Timusk and Bryan Statt, Rep. Prog. Phys. 62 (1999) 61.

[4] S. Chakravarty, R.B. Laughlin, D.K. Morr et al. Phys. Rev. B, 63 (2001) 094503.

[5] Physical properties of high temperature superconductors, ed. D.M. Ginsberg, Vol. I, Singapore, World Scientific(1989); L.P. Regnault, Ph. Bourges and P. Burlet, in Neutron scattering in layered copper-oxide superconductors, ed. A. Furrer, Vol. 20 of Physics and chemistry of materials with low-dimensional structures, Kluger Academic Publishers (1998) p. 85.

[6] G. Grüner, Density waves in solids, Frontiers in physics, vol. 89, Addison-Wesley Publishing Company (1994).

[7] A. Suter, M. Mali, J. Roos et al, Phys. Rev. B 56 (1997) 5542.

[8] B. Grévin, Y. Berthier, and G. Collin, Phys. Rev. Lett. 85 (2000) 1310.

[9] D.J. Derro, K.M. Lang, S.H. Pan et al., Phys. Rev. Lett. 88 (2002) 97002.

[10] J.D. Jorgensen, M.A. Beno, D.G. Hinks et al, Phys. Rev. B 36 (1987) 3608; J.D. Jorgensen, B.W. Veal, A.P. Paulikas et al, Phys. Rev. B 41 (1990) 1863; J.D. Jorgensen, S. Pei, P. Lightfoot et al, Physica C 167 (1990) 571; B.W. Veal, H. You, A.P. Paulikas et al, Phys. Rev. B 42 (1990) 4770.

[11] N.H. Anderson, M. von Zimmermann, T. Frello et al. Physica C 317-318 (1999) 259.

[12] D. de Fontaine, L.T. Wille, and S.C. Moss, Phys. Rev. B 36 (1987) 5709; L.T. Wille and D. de Fontaine, Phys. Rev. B 37 (1988) 2227; D. de Fontaine, M. Asta, G. Ceder et al, Europhys. Lett. 19 (1992) 229.

[13] E. Kaldis, in Handbook on the physics and chemistry of rare earths, Vol. 31, High temperature supercondcutors - II, eds., K.A. Gschneidner, Jr., L. Eyring and M.B. Maple, Elsevier science B. V. (2001) p. 1.

[14] R.J. Cava, B. Batlogg, C.H. Chen et al, Phys. Rev. B 36 (1987) 5719; R.J. Cava, B. Batlogg, C.H. Chen et al, Nature 329 (1987) 423; R.J. Cava, B. Batlogg, S.A. Sunshine et al, Physica C 153 (1988) 560; R.J. Cava, B. Batlogg, K.M. Rabe et al, Physica C 156 (1988) 523; R.J. Cava, A.W. Hewat, E.A. Hewat et al, Physica C 165 (1990) 419; ref. 13].

[15] W.K. Kwok, G.W. Crabtree, A. Umezawa et al. Phys. Rev. B 37 (1988) 106; A.J. Vega, W.E. Farneth, E.M. McCarron et al. Phys. Rev. B 39 (1989) 2322.

[16] M.J. Jurgens, P. Burlet, C. Vettier et al, Physica B 156157 (1989) 846.

[17] J. Zaanen, A.T. Paxton, O.K. Anderson, Phys. Rev. Lett. 60 (1988) 2685; W.E. Pickett, R.E. Cohen, H. Krakauer, Phys. Rev. B 42 (1990) 8764.
[18] H.F. Poulsen, N.H. Andersen, J.V. Andersen et al. Nature 349 (1991) 594.

[19] M. Horvatic, C. Berthier, Y. Berthier et al. Phys. Rev. B 48 (1993) 13848.

[20] H. Yasuoka, T. Shimizu, T. Imai et al. Hyperfine Inter. 49 (1989) 167.

[21] E.E. Tornau, S. Lapinskas, A. Rosengren et al. Phys. Rev. B 49 (1994) 15952.

[22] I. Heinmaa, H. Lutgenmeier, S. Pekker et al. Appl. Magn. Reson. 3 (1992) 689; H. Lutgemeier, I. Heinmaa, A.V. Egorov, Physica Scripta T49 (1993) 137; H. Yasuoka, T. Shimizu, T. Imai et al. Hyperfine Inteactions 49 (1989) 167; E. Lippmaa, E. Joon, I. Heinmaa et al. Physica C 153-155 (1988) 91.

[23] W.W. Warren, Jr., R.E. Walstedt, G.F. Brennert et al. Phys. Rev. Lett. 62 (1989) 1193.

[24] R. Liang, D. Bonn, W.N. Hardy, Physica C 336 (2000) 57.

[25] C.H. Pennington, D.J. Durand, D.B. Zax et al. Phys. Rev. B 37 (1988) 7944; S.E. Barrett, D.J. Durand, C. H. Pennington et al. Phys. Rev. B 41 (1990) 6283.

[26] T. Shimizu, H. Yasuoka, J. Phys. Soc. Japan 57 (1988) 2494.

[27] M. Mali, I. Mangelschots, H. Zimmermann et al. Physica C 175 (1991) 581.

[28] Values for $\gamma_{n}$ and ${ }^{n} Q$ taken from CRC Handbook of Chemistry and Physics 2000-2001, 81'th edition, CRC Press. It should be mentioned that there is a small difference between the $\mathrm{Cu}$ nuclear parameters given in this edition of CRC and the previous ones. We have used the values given in this edition.

[29] H. Yasuoka, in Spectroscopy of Mott Insulator and Correlated Metals, ed. A. Fujimori and Y. Tokura, Solid State Sciences 119, Springer-Verlag, Berlin (1995) p.213.

[30] $T_{2}$ measured for $\mathrm{Cu}(2 \mathrm{~F})$ and $\mathrm{Cu}(2 \mathrm{E})$ are the same.

[31] R. Pozzi, M. Mali, and D. Brinkmann et al, Phys. Rev. B 60 (1999) 9650.

[32] Z. Yamani, B.W. Statt, W.A. MacFarlane et al. in preparation.

[33] Y. Sidis, C. Ultrich, P. Bourges et al. Phys. Rev. Lett. 86 (2001) 4100.

[34] H.A. Mook, P. Dai, F. Dogan, Phys. Rev. B 64 (2001) 012502.

[35] J.E. Sonier, J.H. Brewer, R.F. Kiefl et al. Science 292 (2001) 1692.

[36] C. Stock, W.J.L. Buyers, Z. Tun et al. Phys. Rev. B 66 (2002) 024505.

[37] J.E. Sonier, J.H. Brewer, R.F. Kiefl et al. Phys. Rev. B 66 (2002) 134501.

[38] M.W. Pieper, F. Wiekhorst, T. Wolf, Phys. Rev. B 62 (2000) 1392; S. Schmenn, Untersuchung der Magnetischen Ordnung in Yttrium-Barium-KupferOxid und verwandten Verbindungen mittels Kernresonanz. Dissertation (in german), 1996, Institute für Festkörperforschuang, Jülich.

[39] Vega et al. 15] and T. Imai, private correspondance. Vega 
et al. have the $\mathrm{Cu}(1)_{3}(23 \mathrm{MHz}$ NQR line) relaxation rate about three times faster than for $\mathrm{Cu}(1)_{2}$ at room temperature.

[40] A. Erb, A.A. Manuel, M. Dhalle et al. Solid State Comm. 112 (1999) 245.

[41] R. M. Sternheimer, Phys. Rev. 95 (1954) 736.

[42] E. Straube, D. Hohlwein, F. Kubanek, Physica C 295 (1998) 1.

[43] T. Zeiske, D. Hohlwein, R. Sonntag et al. Physica C 207 (1993) 333.
[44] T. Shimizu, J. Phys. Soc. Jpn. 62 (1993) 779; ibid 62 (1993) 772 .

[45] A. Abragam and B. Bleaney, Electron paramegnetic resonance of transition ions, Clarendon Press, Oxford (1970).

[46] It should also be mentioned that the empirical values 44] of $\gamma_{\infty}$ and $R$ for $\mathrm{Cu}$ ions are obtained for sites with tetragonal symmetry. Hence, these values might not be correct for $\mathrm{Cu}(1 \mathrm{~F})$ site.

[47] G.M. Volkoff, Can. J. Phys. 31 (1953) 820. 